\title{
Comorbidade no transtorno obsessivo-compulsivo
}

Kátia Petribú

Faculdade de Ciências Médicas da Universidade de Pernambuco. Recife, PE, Brasil

\begin{abstract}
Epidemiological and clinical studies indicate that obsessive-compulsive disorder frequently occurs with comorbid disorders. Comorbidity with anxiety and mood disorders, substance abuse or dependence, somatoform, impulse control and tic disorders deserve special attention. In this paper a review of the concept, epidemiological findings, social and occupational functioning, and sequence of comorbid disorders in OCD is presented.
\end{abstract}

Keywords Obsessive-compulsive disorder. Comorbidity.

\section{Introdução}

A associação entre ansiedade e depressão vem sendo descrita na literatura desde o século XIX. Freud, ${ }^{1}$ na publicação "Sobre os critérios para destacar da neurastenia uma síndrome particular intitulada neurose de angústia", afirmou que, embora os casos puros fossem mais marcantes, sintomas de ansiedade ocorriam mais freqüentemente em combinação com aqueles de neurastenia, histeria, obsessão e melancolia.

O termo comorbidade é formado pelo prefixo latino "cum", que significa contigüidade, correlação, companhia, e pela palavra morbidade, originada de "morbus", que designa estado patológico ou doença. Assim, deve ser utilizado apenas para descrever a coexistência de transtornos ou doenças, e não de sintomas.

Feinstein $^{2}$ descreve a comorbidade como "qualquer entidade clínica distinta adicional, que tenha ocorrido ou que venha a ocorrer durante a evolução de um paciente cuja doença índex esteja sob estudo". Em epidemiologia psiquiátrica, a ênfase é dada ao risco relativo (odds ratio): chances de um portador de um determinado transtorno tornar-se mais predisposto a desenvolver outro.

Para haver comorbidade, é importante a relação e a continuidade temporal entre os dois transtornos, que podem surgir simultaneamente ou um preceder o outro. ${ }^{3}$ Nessa mesma linha, Yaryura-Tobias et $\mathrm{al}^{4}$ salientaram que, embora o TOC freqüentemente seja acompanhado por transtornos co-mórbidos relacionados, outras patologias podem ocorrer independentemente em qualquer momento de sua evolução.

\section{As classificações atuais e suas implicações na comorbidade}

As classificações atuais (DSM-IV e CID-10) têm um grande número de categorias a partir do aumento da cobertura diagnóstica e da fragmentação das já existentes e, como adotam critérios operacionais e entrevistas semi-estruturadas, promovem uma "varredura" em praticamente todas as áreas da psicopatologia, aumentando o número de diagnósticos e a probabilidade de associações. A tudo isso alia-se o sistema multiaxial, que permite a presença das síndromes clínicas (eixo I) associadas aos transtornos de personalidade (eixo II) e aos somáticos (eixo III).

Miranda ${ }^{5}$ faz algumas considerações acerca das novas tentativas de interpretação da comorbidade, baseadas nos trabalhos de Wittchen ${ }^{6}$ e Van Praag. ${ }^{7}$ Uma primeira interpretação seria que de fato os pacientes apresentam múltiplos transtornos simultaneamente. Entretanto, isto deve ser questionado, já que as taxas de transtornos co-mórbidos são mais altas que os mesmos transtornos encontrados na população geral, o que não é uma ocorrência comum em medicina, sugerindo que a comorbidade possa ser um artefato dos critérios diagnósticos vigentes.

Uma segunda interpretação seria considerar uma ordem hierárquica na ocorrência dos transtornos, em que uma doença primária predisporia a outra (derivada ou secundária). Outra possibilidade seria de que um fator prévio, como um transtorno de personalidade, precederia e predisporia ao desenvolvimento de outros transtornos. Em muitos pacientes, os transtornos de personalidade (TP) precedem as manifestações psicopatológicas e, em outros, podem originar-se destas. No Brasil, Torres ${ }^{8}$ avaliou 40 portadores de TOC e 40 controles não psiquiátricos e observou a ocorrência de TP em 70\% dos casos e em apenas 15\% dos controles. Apesar de a associação com TP não trazer informações sobre causalidade, isto é, se os traços são primários, secundários ou apenas simultâneos à doença principal, pode-se refletir se essa alta prevalência significa que algumas variáveis biológicas correlacionam-se não só às grandes síndromes psiquiátricas mas também a traços de personalidade. ${ }^{?}$

A quarta interpretação pressupõe que uma alteração neurobiológica poderia provocar, em diferentes regiões e circuitos neuronais, diferentes sintomas ou síndromes. Nesse sentido, Tyrer" denominou de "síndrome neurótica geral" um quadro em que haveria uma predisposição para a manifestação de sintomas ansiosos e depressivos que vêm, vão e se sobrepõem sob a influência de processos biológicos e eventos de vida pouco definidos.

E, por fim, pode-se considerar que as doenças mentais são padrões de reação a um estímulo agressivo, transmitido geneti- 
camente ou adquirido, que provocaria alterações no funcionamento de alguns circuitos neuronais, cuja extensão do comprometimento dependeria de características individuais, da intensidade e da manutenção do estímulo ao longo do tempo.

\section{Aspectos epidemiológicos da comorbidade em TOC}

Os estudos sistematizados têm demonstrado que pelo menos $50 \%$ dos pacientes com TOC apresentam um outro transtorno do eixo $\mathrm{I}^{4,5,10-17}$ e no mínimo $40 \%$ preenchem critérios para um transtorno de personalidade. ${ }^{8}$

A partir de levantamentos epidemiológicos de sete países, Weissman et $\mathrm{al}^{18}$ verificaram que portadores de TOC apresentaram risco maior, variando de 3,8 a 13,5 vezes, de apresentar depressão maior, comparados com aqueles sem TOC, enquanto o risco de apresentar outros transtornos de ansiedade variou de 5,8 a 15,6 vezes. A comorbidade com transtornos de ansiedade foi maior que a de depressão maior.

A presença de comorbidade parece conferir estabilidade e melhorar a validade diagnóstica. Quando os pacientes do ECA que receberam o diagnóstico de TOC foram reentrevistados após 12 meses, constatou-se que a presença de transtornos comórbidos - sobretudo pânico, fobia social e agorafobia - aumentava as taxas de estabilidade diagnóstica. ${ }^{19}$

Depressão é a complicação mais freqüente, sendo a causa mais comum de hospitalização, e tem sido relacionada a gravidade, cronicidade, resposta terapêutica insatisfatória e pior prognóstico. Quanto à comorbidade com transtornos do humor, Perugi et al ${ }^{16,17}$ verificaram que a ocorrência de transtorno do pânico, agorafobia, abuso e dependência de substâncias, assim como início gradual e curso episódico, é mais comum nos pacientes com TOC e trans- torno bipolar que nos portadores de TOC e depressão unipolar.

Yaryura-Tobias \& Neziroglu ${ }^{20}$ consideram que a depressão observada nos pacientes com TOC é mais freqüentemente secundária à incapacitação ocasionada pelos sintomas obsessivocompulsivos do que um transtorno co-mórbido propriamente dito, sugerindo que as cifras de comorbidade sejam menores do que as observadas em outros estudos. Assim, em uma amostra de 409 portadores de TOC, ${ }^{4}$ concluíram que 132 pacientes $(32,2 \%)$ apresentavam no mínimo um outro diagnóstico do eixo I. Destes, $36 \%$ eram portadores de uma patologia do continuum do TOC, e 58\% apresentavam comorbidade.

Miranda ${ }^{5}$ encontrou percentuais altos de comorbidade: a média dos diagnósticos psiquiátricos ao longo da vida nos pacientes com TOC foi de 4,6 e 3,4 diagnósticos no momento atual.

Na Tabela 1 estão resumidos os principais estudos sobre comorbidade no TOC ao longo da vida.

\section{Comorbidade e funcionamento sócio-ocupacional}

A presença de comorbidade interfere na evolução da doença, tornando seu curso mais crônico, com pior prognóstico e resposta ao tratamento. No entanto, poucos estudos compararam tal interferência em outros aspectos do comportamento, como a capacidade adaptativa e o desempenho nas atividades profissionais e sociais. ${ }^{22}$

Na prática clínica, pacientes que apresentam mais de um transtorno são geralmente mais graves do que os portadores de uma patologia isolada. Petribú ${ }^{3}$ não confirmou essa hipótese empírica, tendo encontrado que, quanto à gravidade, os portadores de TOC com ou sem comorbidade foram semelhantes. Miranda ${ }^{5}$ observou que a intensidade grave e moderada do TOC

\begin{tabular}{|c|c|c|c|c|c|c|c|c|c|c|c|}
\hline $\begin{array}{l}\text { Prevalência para } \\
\text { o período de vida }\end{array}$ & $\begin{array}{c}\text { Rasmussen \& } \\
\text { Tsuang } \\
(1986) \\
n=44\end{array}$ & $\begin{array}{c}\text { Eca } \\
\text { (Karno, } \\
1988) \\
n=468\end{array}$ & $\begin{array}{l}\text { Noshirvani } \\
\qquad \begin{array}{l}(1991) \\
n=307\end{array}\end{array}$ & $\begin{array}{c}\text { Rasmussen } \\
\text { \& Eisen } \\
(1992) \\
n=100\end{array}$ & $\begin{array}{c}\text { Torres \& } \\
\text { Smaira } \\
(1993) \\
n=45\end{array}$ & $\begin{array}{l}\text { Kolada } \\
(1994) \\
n=103\end{array}$ & $\begin{array}{c}\text { Petribú } \\
\begin{array}{c}(1996) \\
n=53\end{array}\end{array}$ & $\begin{array}{l}\text { Perugi* } \\
(1997) \\
n=315\end{array}$ & $\begin{array}{c}\text { Miranda } \\
(1999) \\
n=42\end{array}$ & $\begin{array}{c}\text { Yaryura - } \\
\text { Tobias } \\
(2000) \\
n=409\end{array}$ & $\begin{array}{c}\text { Perugi* }^{*} \\
(2001) \\
n=68\end{array}$ \\
\hline Depressão maior & - & $31,7 \%$ & $\begin{array}{c}51 \% \text { mulheres } \\
31 \% \text { homens }\end{array}$ & $67 \%$ & $\begin{array}{c}62 \% \\
\text { (d.menor) }\end{array}$ & $26,9 \%$ & $45,2 \%$ & $34,8 \%$ & $57,1 \%$ & $29,5 \%$ & - \\
\hline Distimia & - & - & - & - & - & $12,4 \%$ & $6,5 \%$ & - & $4,8 \%$ & - & - \\
\hline T. bipolar & - & - & - & - & - & - & - & $15,7 \%$ & $14,3 \%$ & - & $55,8 \%$ \\
\hline $\begin{array}{l}\text { Fobia social } \\
\text { Fobias }\end{array}$ & $18 \%$ & - & - & $18 \%$ & - & - & $16,1 \%$ & - & $40,5 \%$ & - & - \\
\hline específicas & $27 \%$ & $46,5 \%$ & - & $22 \%$ & $49 \%$ & $44,7 \%$ & $6,5 \%$ & - & $52,4 \%$ & $28,8 \%$ & - \\
\hline Agorafobia & $9 \%$ & - & - & $7 \%$ & - & - & $3,2 \%$ & - & $7,1 \%$ & - & - \\
\hline S. Tourette & - & - & - & $7 \%$ & - & - & - & - & $7,1 \%$ & $6,1 \%$ & - \\
\hline Tiques & - & - & - & - & - & - & - & - & $19 \%$ & - & - \\
\hline $\begin{array}{l}\text { T. de pânico } \\
\text { Abuso/ }\end{array}$ & $14 \%$ & $13,8 \%$ & - & $12 \%$ & - & $9,8 \%$ & $6,5 \%$ & - & $14,3 \%$ & $3 \%$ & - \\
\hline dep. de álcool & - & $24,1 \%$ & 8\% homens & $14 \%$ & - & $35,9 \%$ & $6,5 \%$ & - & - & $9,8 \%$ & - \\
\hline $\begin{array}{l}\text { Abuso/ } \\
\text { dep.de } \\
\text { substâncias }\end{array}$ & - & $17,6 \%$ & - & - & - & $26,5 \%$ & - & - & $\begin{array}{c}14,3 \\
\text { (abuso) } \\
14,3 \% \\
\text { (dep) }\end{array}$ & - & - \\
\hline $\begin{array}{l}\text { Esquizofrenia e } \\
\text { tr. psicóticos }\end{array}$ & - & $13,5 \%$ & - & - & - & $11,4 \%$ & - & - & $9,5 \%$ & $10,6 \%$ & - \\
\hline $\begin{array}{l}\text { T. alimentares } \\
\text { Ansiedade de }\end{array}$ & - & - & $12 \%$ mulheres & $17 \%$ & - & $0,4 \%$ & - & - & $14,3 \%$ & $8,3 \%$ & - \\
\hline $\begin{array}{l}\text { separação } \\
\text { Ansiedade }\end{array}$ & $18 \%$ & - & - & - & - & - & - & - & - & - & - \\
\hline generalizada & - & - & - & - & $84 \%$ & - & $29 \%$ & - & $2,4 \%$ & - & - \\
\hline $\begin{array}{l}\text { T. somatoformes } \\
\text { Transtornos do } \\
\text { controle de }\end{array}$ & - & - & - & - & - & $31 \%$ & $16,1 \%$ & - & - & $10,6 \%$ & - \\
\hline impulsos & - & - & - & - & - & - & - & - & $33,2 \%$ & $9,1 \%$ & - \\
\hline
\end{tabular}




\begin{tabular}{|c|c|c|c|c|}
\hline Transtorno & Média idade & Idade (DP) & Mediana & Freqüência \% \\
\hline TDAH & 6,41 & 3,64 & 5,5 & $4,5(n=6)$ \\
\hline Transtorno dismórfico corporal & 13,0 & 5,04 & 12,0 & $6,8(n=9)$ \\
\hline Síndrome de Tourette & 15,13 & 9,65 & 13,0 & $6,1(n=8)$ \\
\hline Transtorno do controle dos impulsos & 16,50 & 6,01 & 16,0 & $9,1(n=12)$ \\
\hline Anorexia nervosa & 17,36 & 7,97 & 14,0 & $8,3(n=11)$ \\
\hline Fobia específica & 18,42 & 9,71 & 16,5 & $28,8(n=38)$ \\
\hline TOC & 18,54 & 10,58 & 17,0 & $-(n=132)$ \\
\hline Esquizofrenia & 21,4 & 11,0 & 18,0 & $10,6(n=14)$ \\
\hline Hipocondria & 21,8 & 11,48 & 17,0 & $3,8(n=5)$ \\
\hline Transtorno de pânico & 24,0 & 7,52 & 24,5 & $3,0(n=4)$ \\
\hline Transtorno afetivo & 24,94 & 10,75 & 20,0 & $29,5(n=39)$ \\
\hline Dependência a substâncias & 28,92 & 12,18 & 27,0 & $9,8(n=13)$ \\
\hline Adaptado de Yaryura-Tobias et al (2000) & & & & \\
\hline
\end{tabular}

foi o principal fator de risco para prejuízo sócio-ocupacional, exercendo maior influência do que o número de diagnósticos psiquiátricos ao longo da vida.

\section{Seqüência temporal de instalação dos transtornos co-mór- bidos ao longo da vida}

Uma linha de pesquisa que tem fornecido importantes contribuições é a sequiência temporal de instalação dos transtornos co-mórbidos ao longo da vida.

Miranda ${ }^{5}$ observou que os transtornos de ansiedade tendem a anteceder a instalação do TOC, enquanto os transtornos do humor acompanham ou, mais freqüentemente, sucedem o TOC. Já os transtornos de abuso/dependência de substâncias em geral surgem após a instalação do TOC, e os do espectro do TOC podem ocorrer em qualquer momento. Yaryura-Tobias et $\mathrm{al}^{4}$ observaram que portadores de TOC apresentam, após sua instalação, risco significativamente maior de desenvolver um transtorno de ansiedade, do humor, de alimentação ou tiques. Esses dados encontram-se resumidos na Tabela 2.

Segundo o ECA, ${ }^{21}$ de cada quatro portadores de TOC, três apresentavam pelo menos um outro diagnóstico psiquiátrico. Fobia específica precedeu o início dos sintomas obsessivos em 75\% dos casos e pânico em 60\%. Entretanto, abuso e dependência de substâncias ocorreram mais freqüentemente após o TOC. Essa seqüência de adoecer pode trazer informações importantes sobre a patogênese desses transtornos.

\section{Tratamento}

Pacientes com TOC e comorbidades psiquiátricas necessitam tratar também os transtornos associados, o que usualmente torna os tratamentos mais prolongados, sobretudo a terapia cognitivo-comportamental (TCC). Muitas vezes é difícil determinar qual dos transtornos deve ser tratado primeiro, devendo a escolha se basear na gravidade e na interferência sobre o funcionamento. Nos pacientes que apresentam impulsividade e agressividade, deve-se considerar a possibilidade de prescrição de neurolépticos, lítio ou carbamazepina.

Na comorbidade com transtorno bipolar, torna-se imperativa a prescrição de estabilizadores do humor e, em alguns casos, a utilização de antipsicóticos atípicos. Nesses casos, o antidepressivo de escolha deve ser da classe dos inibidores seletivos da recaptação de serotonina (ISRS), que somente devem ser prescritos após a estabilização do humor. ${ }^{17}$

Os portadores de TOC e esquizofrenia habitualmente respondem bem a um ISRS ou clomipramina em adição aos neurolépticos. Nos que apresentam comorbidade com tiques ou Tourette, os sintomas respondem de forma mais eficaz à associação de ISRS com neurolépticos naqueles que não são responsivos inicialmente aos ISRS. ${ }^{20}$

\section{Conclusões}

O TOC raramente ocorre sozinho; no entanto, ainda não se pode afirmar se o fenômeno da comorbidade reflete a simultaneidade de entidades diagnósticas distintas ou se decorre da sobreposição de sintomas e síndromes.

O estudo da comorbidade é uma área de grande importância, podendo permitir que relevantes informações sejam obtidas sobre a etiologia e a fisiopatologia dos transtornos mentais e apontar inúmeras perspectivas no desenvolvimento da nosologia e do tratamento dos transtornos associados. Pesquisas clínicas sobre comorbidade e fenomenologia deverão ser críticas para determinar os subtipos dessas entidades heterogêneas e quais os tratamentos mais eficazes para esses casos.

Diante de um paciente com TOC, é importante uma avaliação criteriosa em relação à possibilidade de comorbidade. Em casos considerados refratários, a comorbidade deve ser avaliada, pois certamente trará implicações terapêuticas e prognósticas.

\section{Referências}

1. Freud S. Sobre os critérios para destacar da neurastenia uma síndrome particular intitulada neurose de angústia (1895). Ed. Standard Brasileira. v. III. Rio de Janeiro: Imago Editora; 1976.

2. Feinstein AR. The pre-therapeutic classification of comorbidity in chronic disease. J Chron Dis 1970;23:455-68.
3. Petribú K. Comorbidade em transtorno obsessivo-compulsivo [dissertação]. Recife: Universidade Federal de Pernambuco; 1996.

4. Yaryura-Tobias JA, Grunes MS, Todaro J, McKay D, Neziroglu FA, Stockman R. Nosological Insertion of Axis I Disorders in the etiology of Obsessive-Compulsive Disorder. J Anxiety Dis 2000;14:19-30. 
5. Miranda MA. Transtorno Obsessivo-Compulsivo e Comorbidade: um estudo caso-controle [tese]. São Paulo: Universidade Federal de São Paulo; 1999.

6. Wittchen HU. Critical issues in the evaluation of comorbidity of psychiatry disorders. Br J Psychiatry 1996;168(supl 30):9-16.

7. Van Praag HM. Comorbidity (psycho) analysed. Br J Psychiatry 1996;168(supl 30):129-34.

8. Torres AR. Figura e fundo: um estudo de comorbidade do distúrbio obsessivo-compulsivo e distúrbios da personalidade [tese]. São Paulo: Universidade Federal de São Paulo; 1994.

9. Tyrer P. Classification of Anxiety. Brit J Psychiatry 1984;144:78-83.

10. Karno M, Golding JM, Sorenson SB, Burnham A. The epidemiology of obsessive-compulsive disorder in five US communities. Arch Gen Psychiatry 1988;45:1094-9.

11. Rasmussen $S$, Tsuang M. Clinical characteristics and family history in DSM-III obsessive disorder. Am J Psychiatry 1986;143:317-22.

12. Noshirvani HF, Kasvikis Y, Marks IM, Tsakiris F, Monteiro WO. Gender-divergent aetiological factors in obsessive-compulsive disorder. Br J Psychiatry 1991;158:260-3.

13. Rasmussen S, Eisen JL. The epidemiology and differential diagnosis of obsessive compulsive disorder. J Clin Psychiatry 1992;53(supl 4):4-10.

14. Torres AR, Smaira SI. Características clínicas do distúrbio obsessivocompulsivo: estudo de 45 casos. Rev ABP-APAL 1993;15:44-50.

15. Kolada JL, Bland RC, Newman SC. Obsessive compulsive disorder. Acta Psychiatr Scand 1994;376:24-35.
16. Perugi G, Akiskal HS, Pfanner C, Presta S, Gemignani A, Milanfranchi A et al. The clinical impact of bipolar and unipolar affective comorbidity on obsessive-compulsive disorder. J Affect Disord 1997;46:15-23.

17. Perugi G, Toni C, Akiskal HS. The complex comorbidity between bipolar illness and OCD: clinical implications and treatment outcome. Abstracts of the 2001 Annual Meeting - New Orleans (LA): American Psychiatric Association; 2001. May 5-10.

18. Weissman MM, Bland RC, Canino GJ, Greenwald S, Hwu HG, Lee $\mathrm{CK}$ et al. The cross national epidemiology of obsessive compulsive disorder. J Clin Psychiatry 1994;55(supl 3):5-10.

19. Nelson E, Rice J. Stability of diagnosis of obsessive-compulsive disorder in the Epidemiologic Catchment Area Study. Am J Psychiatry 1997;154:826-31.

20. Yaryura-Tobias JA, Neziroglu FA. Obsessive Compulsive Disorder Spectrum: pathogenesis, diagnosis, and treatment. Washington (DC): American Psychiatric Press; 1997.

21. Regier DA, Burke JD-Jr, Burke KC. Comorbidity of affective and anxiety disorders in the NIMH Epidemiologic Catchment Area Program. In: Maser JD, Cloninger CR, editors. Comorbidity of mood and anxiety disorders. Washington: American Psychiatric Press; 1990. p. 113-22.

22. Newman DL, Moffit TE, Caspi A, Silva PA. Comorbid mental disorders: implications for treatment and sample selection. J Abnorm Psychol 1998;107:305-11.

\section{Correspondência: Kátia Petribú}

Rua Sport Clube do Recife, 280, sala 114 -50070-450 Ilha do Leite, Recife, PE

E-mail: katiapetribu@aol.com 